\title{
NULLITY AND GENERALIZED CHARACTERISTIC CLASSES OF DIFFERENTIAL MANIFOLDS ${ }^{1}$
}

BY

\author{
SIN-LENG TAN
}

\begin{abstract}
Using the Kamber-Tondeur construction of characteristic classes for foliated bundes, the author has given a method for constructing generalized characteristic classes for a differentiable manifold $M$ without imposing conditions on $M$. In particular a vanishing theorem on the manifold $M$ is obtained. The construction is particularly useful if the ordinary characteristic ring $\operatorname{Pont}^{*}(M)$ of the manifold $M$ vanishes much below the dimension of $M$.
\end{abstract}

1. Introduction. The theory of generalized (ordinary and secondary) characteristic classes of a manifold $M$ which is associated with a certain geometric structure is a recent achievement in differential geometry. In contrast to the ordinary characteristic classes, the newly constructed secondary classes carry with them underlying geometric structures. The construction of secondary classes began in a series of papers by Bott [1], Bott and Haefliger [2], Chern and Simons [5], Godbillon and Vey [6], Kamber and Tondeur [7], [8] and others. The idea of the construction is as follows, namely, if the characteristic form $f(K)$ is zero, then the transgression $T f(K)$ of $f(K)$ defines a secondary class in the principal fibre bundle [5]; here $f$ is an invariant polynomial and $K$ is the curvature form. Kamber and Tondeur found the usefulness of the Weil algebra $W(G)$ of a Lie group $G$ in this context. They showed that for a foliated bundle $(P, E,\{\omega\})$ [7], Bott's vanishing theorem [1], when interpreted in the Weil homomorphism [3], $k(\omega)$ : $W(G) \rightarrow A(P)$, gave rise to a new generalized characteristic homomorphism $\Delta_{*}: H\left(W(G, H)_{q}\right) \rightarrow H(M, R)$ where $E$ denotes a foliation on the manifold $M$ of codimension $q,\{\omega\}$ is the family of adapted connections and $W(G, H)_{q}$ denotes the truncated relative Weil algebra [7], [8].

We are able to define generalized characteristic classes on an affine (or Riemannian) manifold $M$ using the Cartan-Kamber-Tondeur map $\Delta_{*}$. We can do this by observing that the affine (or Riemannian) manifold is already foliated by its nullity distribution (\$2). This construction is particularly useful if

Received by the editors February 4, 1976.

AMS (MOS) subject classifications (1970). Primary 57D20, 57D30; Secondary 58A30.

( ${ }^{1}$ ) This paper is based on a part of the author's Ph.D. Thesis (1974) written at the University of Illinois, under the direction of Professor F. Kamber. For the unexplained notions, see [8].

- American Mathematical Society 1978 
the ordinary characteristic ring of the manifold $M$ vanishes much below the dimension of the manifold.

We show that if $\nabla$ is a connection on $M$ compatible with a $G$-structure such that $N$ is the (possibly singular) nullity distribution of $\nabla$, then we have the following generalized characteristic homomorphism:

$$
\Delta_{*}: H\left(W(G, H)_{[(n-\mu) / 2]}\right) \rightarrow H(M, R),
$$

where $H$ denotes an $H$-reduction of the $G$-principal fibre bundle $P$ and $\mu$ is the minimum index of the nullity distribution $N$ (Theorem 4.1). In particular, we have Pont ${ }^{*}(M)=0$, for ${ }^{*}>n-\mu$ (Theorem 4.2). This vanishing theorem does not hold for integral characteristic ring $\operatorname{Pont}^{*}(M, Z)$. Moreover, we show by an example that the vanishing theorem does not follow from the underlying fibred structure of the manifold.

We observe that Bott's vanishing theorem not only holds for the normal bundle of the nullity distribution, but also for the nullity distribution itself. Finally, there exist examples that the constructions do produce nontrivial secondary classes.

2. Nullity distributions. Let $(M, \nabla)$ be an affine manifold, namely a differential manifold $M$ on which $\nabla$ is the connection in the tangent bundle $T M$. The curvature tensor $R$ can be considered as a bundle map $R: T M \rightarrow$ Hom $(T M$, End $(T M))$. The nullity distribution $N$ [4] can be defined as the kernel of the map $R$. The nullity space at $m \in M$ is the space $N_{m}=\{X \in$ $\left.T M_{m} \mid R(X,-)=0\right\}$. The dimension $\mu(m)$ of $N_{m}$ is called the index of nullity at $m$.

Results obtained by Chern and Kuiper [4] and Maltz [11] on Riemannian nullity distributions can be generalized to affine manifolds [13]. In particular, it is shown that the presheaf of $N$ is involutive. Thus, if $N$ is nonsingular, then $N$ constitutes a natural foliation on $M$ which foliates the manifold into a flat part and a nonflat part.

Moreover, it is shown in [13] that for an almost free action of a compact Lie group $G$ on a manifold $M$, then there exists an affine structure on $M$ with nullity index at least the dimension of $G$. Also for Riemannian symmetric spaces of compact or noncompact type, their nullity distributions are zero.

3. Foliated bundles and generalized characteristic classes. Let $P(M, G, \pi)$ be a principal bundle, and $E$ be a foliation on $M$. Let $\Omega$ be the set of annihilator 1-forms of $E$. Since $E$ is integrable, $\Omega$ generates a differential ideal in the exterior algebra $A(M)$ of differential forms on the manifold $M$.

Consider now the Weil homomorphism of differential graded algebras,

$$
k(\omega): W(G) \rightarrow A(P)
$$


defined by a connection $\omega: A^{1}(P) \rightarrow$ \& in the principal bundle $P(M, G, \pi)$ [3], [8]. Here $W(G)$ is the Weil algebra which is the tensor product of the exterior algebra $\wedge \mathbb{S}^{*}$ and the symmetric algebra $S\left(\mathbb{S}^{*}\right.$. The isomorphism $\wedge^{1} \mathbb{S}^{*} \rightarrow S^{1} \mathbb{S}^{*}$ is denoted by $\alpha \rightarrow \tilde{\alpha}$. The homomorphism $k(\omega)$ induces on the subalgebra of invariant polynomials $I(\mathbb{B}) \subset W(G)$ the Chern-Weil homomorphism.

Since $\omega$ defines $k(\omega)$ uniquely and vice versa, we consider $k(\omega)$ as a connection on $P$. The curvature of the connection is the map $K(\omega): \wedge^{1}\left(S^{*} \rightarrow\right.$ $A^{2}(P)$ defined by $d_{P} k(\omega)-k(\omega) d_{\wedge}$.

There are canonical decreasing filtrations on $W(G)$ and $A(P)$ defined by

$$
\begin{aligned}
F^{2 p} W(G) & =S^{p}\left(\mathbb{S}^{*}\right) \cdot W(G), \quad F^{2 p-1} W=F^{2 p} W \\
F^{p} A(P) & =\Omega^{p} \cdot A(P) .
\end{aligned}
$$

A connection $k(\omega)$ (or simply $\omega$ ) on $P$ is said to be $E$-adapted (respectively basic) if $K(\omega) \beta(X, Y)=0$ (respectively $K(\omega) \beta(X,-)=0$ ) for $X, Y \in E$ and $\beta \in$ (ङ). Here we identify the basic elements $A(P)_{G}$ in $A(P)$ with $A(M)$ [3]. Two adapted connections $\omega_{1}$ and $\omega_{2}$ in $P$ are said to be equivalent if

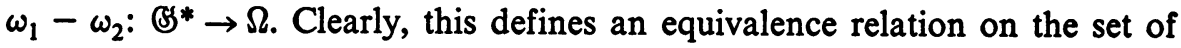
adapted connections. The filtrations defined in (3.2) are preserved by $E$ adapted connections. The fact that $F^{p} A(P)=0$ for $p>q$ implies that $k(\omega) F^{2(q+1)}=0$ and, in particular, $k(\omega) I(\mathbb{S})^{2(q+1)}=0$. This is Bott's vanishing theorem [1]. Moreover this fact gives rise to secondary classes [5], [6], [8].

Following Kamber and Tondeur [7], a foliated bundle is a triple $(P, E,\{\omega\})$ such that $E$ is a foliation on $M$, and $\{\omega\}$ is an equivalence class of adapted connections on $P$ with respect to the foliation $E$.

Let $H$ be a closed subgroup of $G$. Then we have the following commutative diagram:

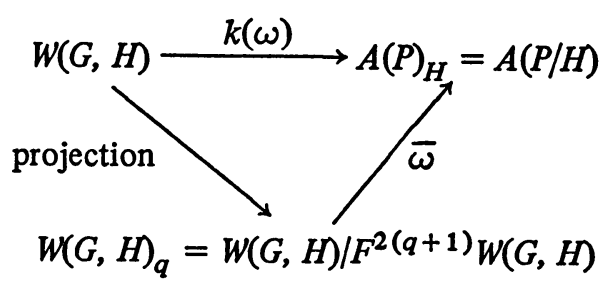

where $W(G, H)$ denotes the $H$-basic elements in $W(G) . W(G, H)_{q}$ is called the truncated relative Weil algebra. Hence, if $s: M \rightarrow P / H$ is an $H$-reduction of the principal bundle, then we have 


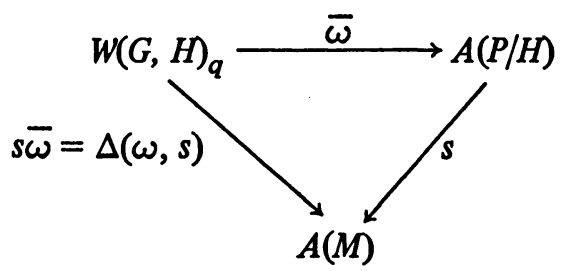

Theorem 3.1 (KAMber AND Tondeur [8]). Let $P(M, G, \pi)$ be a principal bundle with an $H$-reduction s: $M \rightarrow P / H$. Let $(P, E,\{\omega\})$ be a foliated bundle, and let $\omega$ be an adapted connection in $\{\omega\}$. Then there exists a generalized characteristic homomorphism

$$
\Delta_{*}: H\left(W(G, H)_{q}\right) \rightarrow H(M, R)
$$

such that $\Delta_{*}=\Delta(\omega, s)_{*}$ is independent of the choice of adapted connection $\omega$ in $\{\omega\}$ and depends only on the homotopy class of $s$. Moreover if $\omega$ is basic, the homomorphism $\Delta_{*}$ is the map

$$
\Delta_{*}: H\left(W(G, H)_{[q / 2]}\right) \rightarrow H(M, R) .
$$

The Kamber-Tondeur construction above contains, besides the usual characteristic classes of $P$, secondary invariants [8], [9]. We shall use Theorem 3.1 to construct some secondary invariants on affine manifolds naturally.

4. Generalized characteristic classes. Let $(M, \nabla)$ be an affine manifold. Let $\omega$ be the corresponding connection form of $\nabla$ in $F(M)$, the frame bundle of $M$. Let $P(M, G, \pi)$ be a $G$-reduction of the frame bundle. We say that the connection $\nabla$ is compatible with the $G$-reduction if $\omega$ reduces to a connection in $P$. We denote the induced connection in $P$ again by $\omega$. We note that $K(\omega) \alpha\left(X^{*}, Y^{*}\right)=0$ if and only if $R(X, Y)=0$, where $X^{*}$ and $Y^{*}$ are the horizontal lifts of $X$ and $Y$ in $\mathfrak{X}(P)=A^{1}(P)$ and $\alpha \in \Lambda^{1}(s)$.

Recall that $N_{m}$ is the nullity space at $m \in M$. The index of nullity at $m$ is denoted by $\mu(m)$ which is the dimension of the nullity space $N_{m}$. Let $\mu=\operatorname{minimum}_{m \in M} \mu(m)$. From the definition of $N_{m}$, we have $R(X,-)=0$ for all $X \in N_{m}$.

THEOREM 4.1. Let $(M, \nabla)$ be an affine manifold. If $\nabla$ is compatible with a $G$-structure $P$, then we have a characteristic homomorphism

$$
\Delta_{*}: H\left(W(G, H)_{[q / 2]}\right) \rightarrow H(M, R), \quad q=n-\mu,
$$

where $H$ denotes an $H$-reduction of the principal $G$-bundle $P$.

Proof. We first show that $k(\omega): W(G) \rightarrow A(P)$ maps $F^{2([q / 2]+1)} W(G)$ into zero. Since $k(\omega)$ is an algebra homomorphism, it suffices to show that $k(\omega)$ maps elements of type $\tilde{\alpha}_{1} \tilde{\alpha}_{2} \ldots \tilde{\alpha}_{k}$ in $S^{k}$ (ङङ*) into zero, where $\tilde{\alpha}_{i} \in S^{1}\left(S^{*}\right)$, and $k \geqslant[q / 2]+1$. Since 


$$
k(\omega)\left(\tilde{\alpha}_{1} \tilde{\alpha}_{2} \ldots \tilde{\alpha}_{k}\right)=K(\omega) \alpha_{1} K(\omega) \alpha_{2} \ldots K(\omega) \alpha_{k}
$$

is a horizontal $2 k$-form on $P, k(\omega)\left(\tilde{\alpha}_{1} \ldots \tilde{\alpha}_{k}\right)=0$ if and only if

$$
k(\omega)\left(\tilde{\alpha}_{1} \ldots \tilde{\alpha}_{k}\right)\left(X_{1}^{*}, \ldots, X_{2 k}^{*}\right)=0
$$

for all $X_{i} \in T M_{m}$. Since $\mu=\operatorname{Min} \mu(m)$, at each point of $m \in M$, we see that $2 k>n-\mu(m)$. At the point $m \in M$, let

$$
\left\{Y_{1 m}, Y_{2 m}, \ldots, Y_{n-\mu(m)}, Z_{1 m}, \ldots, Z_{\mu(m) m}\right\}
$$

be a basis for $T M_{m}$ such that $Y_{i m} \notin N_{m}$, and $Z_{j m} \in N_{m}$. Since $k-1>$ [ $(n-\mu) / 2]$, either the $2 k$-vectors $X_{1}, \ldots, X_{2 k}$ are linearly dependent, or they must be linear combinations of the $Y_{i m}$ 's plus at least one vector from the $Z_{j m}$ 's. In the first case, $k(\omega)\left(\tilde{\alpha}_{1} \ldots \tilde{\alpha}_{k}\right)=0$ automatically, and in the second case, we have

$$
\begin{aligned}
k(\omega)\left(\tilde{\alpha}_{1}\right. & \left.\ldots \tilde{\alpha}_{k}\right)\left(X_{1}^{*}, \ldots, X_{2 k}^{*}\right) \\
& =\sum \varepsilon_{\sigma} K(\omega) \alpha_{1}\left(X_{\sigma(1)}^{*} X_{\sigma(2)}^{*}\right) \ldots K(\omega) \alpha_{k}\left(X_{\sigma(2 k-1)}^{*}, X_{\sigma(2 k)}^{*}\right)
\end{aligned}
$$

where $\varepsilon_{\sigma}$ denotes the sign of the permutation $\sigma$ such that $\sigma(1)<$ $\sigma(2), \ldots, \sigma(2 k-1)<\sigma(2 k)$. Without loss of generality, we assume $X_{1}=$ $Z_{1 m}$. Then it is clear that each term on the right-hand side of (4.1) vanishes, since one of $K(\omega) \alpha_{i}\left(X_{\sigma(2 i-1)}^{*}, X_{\sigma(2 i)}^{*}\right)$ must be $K(\omega) \alpha_{i}\left(Z_{1 m}^{*}, X_{\sigma(2 i)}^{*}\right)$, which is zero from the preceding remark. Hence $k(\omega)\left(\tilde{\alpha}_{1} \ldots \tilde{\alpha}_{k}\right)=0$. Now it follows from the proof of Theorem 3.1 [8] that we have such a characteristic homomorphism. This completes the proof. Q.E.D.

Theorem 4.2. Let $(M, \nabla)$ be an affine manifold. Then $\operatorname{Pont}^{*}(M)=0$ for $* n-\mu$.

Proof. This is immediate from the proof of the above theorem by putting $P=F(M), H=G=\mathrm{GL}(n, R)$. Q.E.D.

If the index of nullity is equal to $\mu$, then locally, the manifold $M$ splits off a $\mu$-dimensional Euclidean factor. From the homological point of view, the manifold $M$ behaves as if it would split off an Euclidean factor globally. In the case of (globally) symmetric spaces, this is indeed the situation [13, Theorem 4.6]. However, at the end of this section, we are able to construct a locally symmetric space $M$ which has the vanishing property of Theorem 4.2 and which is not a submersion over a second manifold of dimension $n-\mu$.

If the ordinary characteristic ring Pont $(M)$ of the manifold $M$ in Theorem 4.2 vanishes much below the dimension of $M$, then the ordinary characteristic invariants give us very little information about the manifold. However, the generalized characteristic homomorphism $\Delta_{*}$ in Theorem 4.1 defines a larger set of invariants. It contains not only the ordinary characteristic invariants but also the secondary invariants. Consequently, the secondary invariants which 
we have constructed actually supplement the ordinary characteristic invariants of the manifold $M$. They. are finer invariants than the ordinary characteristic classes. In fact they depend not only on the bundle in question, but also on the foliated structure on the bundle. They may be nontrivial even if the underlying bundle is trivial. We shall discuss these secondary invariants in more detail in $\$ \S 5$ and 6.

COROLlARY 4.3. If $N$ is a (nonsingular) nullity foliation, then Pont* $(M)=0$ for ${ }^{*}>$ codimension of $N$.

It turns out that Theorem 4.2 is the strongest such general vanishing theorem we can get. There are many $4 n$-dimensional manifolds $M$ such that Pont ${ }^{4 n}(M) \neq 0$; here the minimum index $\mu$ of $\mu(x)$ is 0 . This theorem is not true for integral cohomology.

EXAMPLE 4.1. In [12], Pasternack defined an almost free $S^{1}$ (the circle group) action on a 4-dimensional manifold $M$ which is a real blow up of $P_{2}(C)$. He shows that there exists a nontrivial Pontrjagin class in Pont ${ }^{4}(M, Z)$. From a remark in $\$ 2$ the nullity index is at least 1 . Thus, this serves as a counterexample of Theorem 4.2 for integral cohomology.

Our next example shows that the vanishing of the characteristic ring Pont $^{*}(M)=0, *>n-\mu$, in Theorem 4.2 does not come from the underlying geometric structure on $M$. Namely, if the manifold $M$ is a fibration $f: M \rightarrow N$ over another manifold $N$ ( $N$ of dimension $n-\mu$ ) such that ker $f \subset T M$ gives us the nullity distribution which is a flat subbundle of $T M$ by itself, then we have $T M \cong \operatorname{ker} f \oplus f^{*}(T N)$, which implies that $\operatorname{Pont}^{i}(M)=f^{*} \operatorname{Pont}^{i}(T N)=0, i>n-\mu$. We construct a locally symmetric space below, and show that the vanishing of the characteristic classes in Pont $(M)$ does not actually result from a fibration.

ExAMPLe 4.2. Consider the $2 n$-dimensional complex projective space $P_{2 n}(C)$. The manifold $P_{2 n}(C)$ is a symmetric space which is isomorphic to $U(2 n+1) /(U(2 n) \times U(1))$, where $U(2 n+1), U(2 n)$ and $U(1)$ are the unitary groups. The involutive automorphism $\sigma$ of $U(2 n+1)$ is given by setting $\sigma(a)=$ sas $^{-1}$, where

$$
s=\left(\begin{array}{cc}
I_{2 n} & 0 \\
0 & -1
\end{array}\right)
$$

and $I_{2 n}$ is the identity matrix rank $2 n$. The canonical decomposition of the Lie algebra is given by $\mathbb{S}=3+\mathfrak{M}$, where $\mathbb{S}=u(2 n+1)$ : all skewHermitian matrices of degree $2 n+1,3=\left\{u(2 n)+u(1)=\left(\begin{array}{ll}x & 0 \\ 0 & \lambda\end{array}\right), \lambda+\bar{\lambda}=\right.$ $0, x \in u(2 n)\}$, and $\mathfrak{M}=\left\{\left(\begin{array}{c}0 \\ -\xi\end{array}\right), \xi \in C^{2 n}\right\}$.

Let $D$ be the canonical $U(2 n+1)$-(left) invariant connection on $P_{2 n}(C)$. Then the 3 component $\omega$ of $\&$ with respect to the decomposition $\&=3+\mathfrak{M}$ defines the corresponding $U(2 n+1)$-invariant connection in the principal 
$U(2 n) \times U(1)$ bundle $U(2 n+1) \rightarrow P_{2 n}(C)$.

Next, we regard the $2 n+1$-dimensional Euclidean space $R^{2 n+1}$ as a symmetric space. Let $M$ be the product $P_{2 n}(C) \times R^{2 n+1}$. Then it is clear that $M \cong U(2 n+1) \times R^{2 n+1} / U(2 n) \times U(1) \times\{0\}$. Thus, the manifold $M$ becomes a symmetric space with respect to the involutive automorphism $\bar{\sigma}$ of $U(2 n+1) \times R^{2 n+1}$ given by $\bar{\sigma}(a, v)=(\sigma(a),-v)$, for $a \in U(2 n+1)$, $v \in R^{2 n+1}$. The canonical $U(2 n+1) \times R^{2 n+1}$-invariant connection $\bar{\nabla}$ on $M$ is given by the connection form $\bar{\omega}$ in the principal bundle $U(2 n+1) \times R^{2 n+1} \rightarrow M$ by $\bar{\omega}(x, v)=(\omega(x), 0)$, for $x \in \mathbb{S}, v \in R^{2 n+1}$. The nullity distribution of the symmetric space $P_{2 n}(C)$, which is of compact type is zero [13]. It follows that the nullity distribution of $M$ is $M \times R^{2 n+1}$.

Let $v_{1}, v_{2}, \ldots, v_{2 n+1}$ be any basis for $R^{2 n+1}$. Let $L$ be the subgroup of $R^{2 n+1}$ generated by $v_{1}, v_{2}, \ldots, v_{2 n+1}$ :

$$
L=\left\{\sum_{i=0}^{2 n+1} m_{i} v_{i} ; m_{i} \text { integers }\right\} .
$$

The action of $L$ on $R^{2 n+1}$ is properly discontinuous and the quotient manifold $R^{2 n+1} / L$ is a torus. Let $T^{2 n+1}=U(1) \times U(1) \times \cdots \times U(1)$ be the maximal torus in $U(2 n+1)$. Let $\gamma: L \rightarrow T^{2 n+1}$ be any homomorphism such that $\gamma(L)$ is dense in $T^{2 n+1}$. Let $\Gamma=\{(\gamma(v), v): v \in L\}$. It is clear that $\Gamma$ is a discrete subgroup of $U(2 n+1) \times R^{2 n+1}$. The induced $\Gamma$ action on $M, \psi: \Gamma \times M \rightarrow M$, is given by

$$
\psi((\gamma(v), v),(\bar{g}, u))=(\overline{\gamma(v) g}, v+u),
$$

for $(\gamma(v), v) \in \Gamma,(\bar{g}, u) \in M$, and $\bar{g}$ an element in $P_{2 n}(C)$. It is clear that the action $\psi$ on $M$ is properly discontinuous. Hence, the left coset space $\Gamma \backslash M$ is a $(6 n+1)$-dimensional compact manifold such that $M \rightarrow \Gamma \backslash M$ is a covering. Since the connection $\bar{\nabla}$ on $M$ is left $U(2 n+1) \times R^{2 n+1}$ invariant, it induces a connection $\nabla$ on $\Gamma \backslash M$. It is clear that, with respect to the induced connection $\nabla, \Gamma \backslash M$ becomes a locally symmetric space. Since the nullity distribution of $M$ is equal to $M \times R^{2 n+1}$, the nullity distribution $N$ of $\Gamma \backslash M$, which is a regular foliation of $\Gamma \backslash M$ [13], is also of dimension $2 n+1$. Hence Pont ${ }^{*}(\Gamma \backslash M)=0,{ }^{*}>4 n$ (Theorem 4.2). Although $M$ is a fibration over $P_{2 n}(C)$ which has flat leaves of the nullity distribution defined by $\bar{\nabla}$ as its fibres, the manifold $\Gamma \backslash M$ is not a submersion over $\gamma(L) \backslash P_{2}(C)$, because $\gamma(L) \backslash P_{2}(C)$ is not even a manifold $(\gamma(L)$ does not act properly discontinuous on $P_{2 n}(C)$ ). Thus, we have a family of examples such that the vanishing of the characteristic ring does not come from the underlying geometric structure.

We note that $\operatorname{Pont}^{4 n}(\Gamma \backslash M) \neq 0$. Since $M \stackrel{\pi}{\rightarrow} \Gamma \backslash M$ is a covering, we have 
$\pi^{*}(T(\Gamma \backslash M))=T(M)$. Since $M$ and $P_{2 n}(C)$ are of the same topological type, we have Pont* $(M)=\operatorname{Pont}^{*}\left(P_{2 n}(C)\right)$. Let $P_{n}(N)$ denote the $n$th Pontrjagin class of a manifold $N$. It is well known that

$$
P_{n}(M)=P_{n}\left(P_{2 n}(C)\right)=(-1)^{n}(2 n+1) \eta \neq 0,
$$

where $\eta$ is the fundamental form of $P_{2 n}(C)$. By the naturality of characteristic classes, we have $\pi^{*} P_{n}(\Gamma \backslash M)=P_{n}(M) \neq 0$. This proves that Pont ${ }^{4 n}(\Gamma \backslash M)$ $\neq 0$.

More generally, we can consider a symmetric space $G / H$ such that $H$ is compact and uniform. Then the manifold $M=G / H \times R^{k} \cong G \times R^{k} / H$ is a symmetric space whose nullity bundle contains the subbundle $M \times R^{k}$. Let $L$ be any infinite discrete subgroup in $R^{k}$, and let $\gamma: L \rightarrow G$ be any homomorphism such that $\gamma(L)$ has accumulation points in $G$ (that is, $\gamma(L)$ does not act properly discontinuously on $G / H$ ). We define the subgroup $\Gamma \subset G \times R^{k}$ as above. Then the double quotient $\Gamma \backslash G \times R^{k} / H$, which is locally symmetric, will have the property that the previous example has, namely the vanishing property does not come from a submersion.

5. Riemannian nullity foliated bundles. Let $(M, g)$ be a Riemannian manifold with the metric $g$. Let $\nabla$ be the Riemannian connection on $(M, g)$. We assume from now on that the nullity foliation $N$ is nonsingular and of codimension $q$. Under the metric $g$, we have the following split exact sequence,

$$
0 \longrightarrow R_{\pi}^{\stackrel{i}{\longrightarrow} T M} \underbrace{\stackrel{p}{\longrightarrow} Q}_{j}=N^{\perp} \longrightarrow 0
$$

where $Q$ is the normal bundle of $N$ and is given by $N^{\perp}=\{X \in$ $T M \mid g(X, Y)=0$ for all $Y \in N\}$.

Using the Riemannian connection $\nabla$ in $T M$, we are able to define two induced connections $D$ and $\bar{D}$ on the nullity bundle $N$ and its normal bundle $Q$, respectively, as follows:

$$
\begin{array}{ll}
D_{X} Y=\pi \nabla_{X} Y, & \text { for } X \in \mathfrak{X}(M), Y \in \Gamma(N), \\
\bar{D}_{X} Z=p \nabla_{X} Z, & \text { for } X \in \mathfrak{X}(M), Z \in \Gamma(Q) .
\end{array}
$$

Proposirion 5.1. The induced connections $D$ and $\bar{D}$ in $N$ and $Q$, respectively, are $N$-adapted connections which are basic. Moreover they are metric with respect to the induced metrics in $N$ and $Q$.

Proof. It is easy to show that the connections are $N$-adapted metric connections. Next we show that $\bar{D}$ is basic. It suffices to show that $\bar{R}(X, Y)=$ 0 for $X \in \Gamma(N)$ and $Y \in \Gamma(Q)$. Note that $\nabla_{X} W \in N$ for $X, W \in N$ since 
the leaves of the foliation $N$ are totally geodesic in $M$ [10, $\S 8$ of Chapter 7]. We have, therefore,

$$
\begin{aligned}
\bar{R}(X, Y) Z & =\bar{D}_{X} \bar{D}_{Y} Z-\bar{D}_{Y} \bar{D}_{X} Z-\bar{D}_{[X, Y]} Z, \quad Z \in \Gamma(Q), \\
& =p \nabla_{X} p \nabla_{Y} Z-p \nabla_{Y} p \nabla_{X} Z-p \nabla_{[X, Y]} Z \\
& =p \nabla_{X} \nabla_{Y} Z-p \nabla_{Y} \nabla_{X} Z+p \nabla_{Y} \pi \nabla_{X} Z-p \nabla_{[X, Y]} Z \\
& =p R(X, Y) Z+p \nabla_{Y} \pi \nabla_{X} Z=p \nabla_{Y} \pi \nabla_{X} Z
\end{aligned}
$$

because $R(X, Y) Z=0$. Hence

$\bar{R}(X, Y) Z=0 \Leftrightarrow p \nabla_{Y} \pi \nabla_{X} Z=0 \Leftrightarrow g\left(\nabla_{Y} \pi \nabla_{X} Z, W\right)=0$, for $W \in \Gamma(Q)$.

Since

$$
\begin{aligned}
& 0=Y g\left(\pi \nabla_{X} Z, W\right)=g\left(\nabla_{Y} \pi \nabla_{X} Z, W\right)+g\left(\pi \nabla_{X} Z, \nabla_{Y} W\right), \quad \text { and } \\
& 0=X g\left(Z, \pi \nabla_{Y} W\right)=g\left(\nabla_{X} Z, \pi \nabla_{Y} W\right)+g\left(Z, \nabla_{X} \pi \nabla_{Y} W\right),
\end{aligned}
$$

we therefore have

$$
\begin{aligned}
\bar{R}(X, Y) Z & =0 \Leftrightarrow g\left(\pi \nabla_{X} Z, \nabla_{Y} W\right) \\
& =0 \Leftrightarrow g\left(\nabla_{X} Z, \pi \nabla_{Y} W\right)=0 \Leftrightarrow g\left(Z, \nabla_{X} \pi \nabla_{Y} W\right)=0 .
\end{aligned}
$$

By the remark above, $\nabla_{X} \pi \nabla_{Y} W \in \Gamma(N)$ and the fact that $Z \in \Gamma(Q)$, we actually have $g\left(Z, \nabla_{X} \pi \nabla_{Y} W\right)=0$, which shows that $\bar{D}$ is a basic $N$-adapted connection in $Q$. Similarly, $\bar{D}$ is a basic $N$-adapted connection in $N$. Q.E.D.

Theorem 5.2. Pont ${ }^{*}(N)=0$ and $\operatorname{Pont}^{*}(Q)=0$ for $^{*}>q$.

PROof. It follows from the above theorem that the curvature two forms of the connections lie in $\Omega^{2}$. Q.E.D.

Hence, for a Riemannian manifold $(M, g)$, all three vector bundles in (5.1) are $N$-foliated bundles. Moreover, the foliated bundles are all basic with respect to the (induced) metric connections defined on them. For an affine manifold $(M, \nabla), \bar{D}$ is a well-defined connection in the normal bundle $Q$ if $\nabla_{X} Y \in N$ for $X, Y \in N$. This means that the curvature tensor of $\nabla$ is parallel with respect to the nullity foliation $N$. Even in this situation, $D$ and $\bar{D}$ define $N$-adapted connections which are not necessarily basic. Hence we have only

$$
\operatorname{Pont}^{*}(N)=0 \text { and } \operatorname{Pont}^{*}(Q)=0 \text { for }{ }^{*}>2 q \text {. }
$$

The fact that Pont* $(Q)=0$ for ${ }^{*}>2 q$ follows from Bott's vanishing theorem [1] without using the induced connection $\bar{D}$.

The Bott canonical $N$-adapted connection $\bar{\nabla}$ on $Q$ is characterized by putting $\bar{\nabla}_{X} s=p[X, Y]$ for $X \in \Gamma(E), s \in \Gamma(Q)$ and $Y \in(M)$ such that $p(Y)=s$.

Proposition 5.3. The two adapted connections $\bar{D}$ and $\bar{\nabla}$ in $Q$ belong to the 
same equivalence class of $N$-adapted connection if and only if $p \nabla_{Y} X=0$ for all $Y \in \Gamma(Q)$ and $X \in \Gamma(N)$.

PROOF. It follows from routine calculation.

6. Nullity foliations and generalized characteristic classes. Let $(M, \nabla)$ be an affine manifold with nullity foliation $N$. Then we have the following split exact sequence:

$$
0 \rightarrow N \stackrel{i}{\rightarrow} T M \stackrel{p}{\rightarrow} Q \rightarrow 0 .
$$

Hence $T M \cong N \oplus Q$. Therefore the structure group of $T M$ (which is $\mathrm{GL}(n, R))$ can be reduced to $O(k) \times O(n-k)$, where $k$ is the dimension of $N$.

THEOREM 6.1. Let $(M, \nabla)$ be an affine manifold which has nullity foliation $N$ of codimension $q=n-k$. Then there exists a generalized characteristic homomorphism,

$$
\Delta_{*}: H^{\cdot}\left(W(\mathrm{GL}(n, R), O(k) \times O(q))_{[q / 2]}\right) \rightarrow H^{\cdot}(M, R),
$$

such that $\Delta_{*}$ depends only on the equivalence class of adapted connections determined by the given basic connection $\nabla$, and depends only on the homotopy class of the reduction of the structure group of $M$ to $O(k) \times O(q)$.

Proof. Since the affine connection is already a basic $N$-adapted connection in $T M$ and since there exists such a $O(K) \times O(n-k)$-reduction, the theorem now follows from Theorem 3.1. Q.E.D.

The above proposition gives a natural way to construct secondary classes for an affine manifold $(M, \nabla)$. The only assumption we imposed here is that the nullity foliation should be nonsingular. In the next section, we present an example constructed by Kamber and Tondeur [8], where if the affine manifold is flat, that is, if $N=T M$, then there are nontrivial secondary invariants which can be constructed as in the above proposition.

If the affine connection is a metric connection (with respect to a metric $g$ ) such that locally the metric $g$ splits into a direct product metric of $N$ and $Q=N$, then the metric $g$ gives rise to an $O(k) \times O(n-k)$-reduction $P^{\prime}$ of the frame bundle $F(M)$ such that the given metric connection can be reduced to a connection $\omega^{\prime}$ in $P$. Let $i: O(k) \times O(n-k) \rightarrow \mathrm{GL}(n, R)$ be the inclusion map. Then $W(i): W(\mathrm{GL}(n, R)) \rightarrow W(O(k) \times O(n-k))$ is the restriction which is a $O(k) \times O(n-k)-D G$ homomorphism. Since the connection $\omega$ in $F(M)$ can be reduced to $P^{\prime}$, we find that $\Delta_{*}$ in Theorem 6.1 factorizes through $W(O(k) \times O(n-k), O(k) \times O(n-k))_{[q / 2]}$ as follows: 


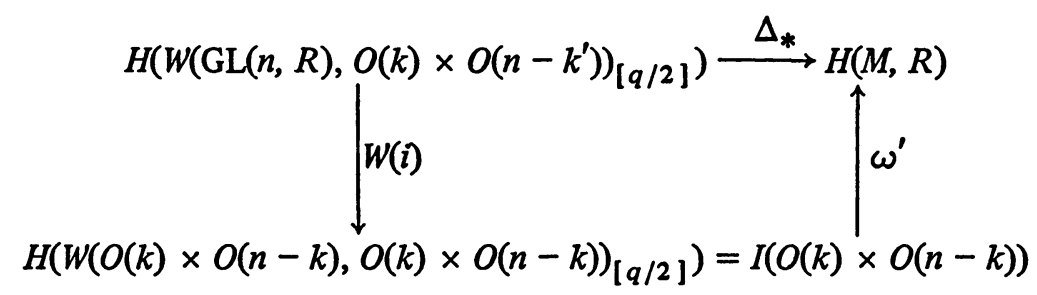

Here $\omega^{\prime}$ is the usual Chern-Weil homomorphism. Hence, the generalized characteristic homomorphism $\Delta_{*}$ does not give rise to secondary classes. Consequently, in Theorem 6.1, the nonzero secondary invariants which are contained in the image of $\Delta_{*}$ are obstructions to the existence of a metric $g$ such that locally $g$ splits into a direct product of the nullity $N$ and its normal bundle $Q$.

For Riemannian manifold, we have

THEOREM 6.2. (i) Let $(M, g)$ be a Riemannian manifold. Then (6.2) becomes

$$
\Delta_{*}: H\left(W(O(n), O(k) \times O(q))_{[q / 2]}\right) \rightarrow H(M) .
$$

(ii) If there exists an $H$-reduction of the structure group $O(k)$ of the nullity foliation, then there exists the generalized characteristic map for $N$ :

$$
\Delta_{N^{*}}: H\left(W(O(k), H)_{[q / 2]}\right) \rightarrow H(M) .
$$

(iii) Similarly, on the normal bundle, we have

$$
\Delta_{Q^{*}}: H\left(W\left(O(q), H^{\prime}\right)_{[q / 2]}\right) \rightarrow H(M)
$$

if there exists an $H^{\prime}$-reduction for the normal bundle $Q$.

Proof. This follows from Theorems 3.1, 6.1 and the fact that all the adapted connections involved are basic metric connections. Q.E.D.

7. Examples. The two examples which we will discuss here were constructed by Kamber and Tondeur [8], [9]. These examples show that a manifold foliated by its nullity foliation $N$ actually gives rise to secondary classes.

Let $(M, \nabla)$ be a connected flat affine manifold. Then from Theorem 6.1, we have

$$
\Delta_{*}: H\left(W(\mathrm{GL}(n, R), O(n))_{0}\right) \rightarrow H(M) .
$$

It is shown in [8] that

$$
W(\mathrm{GL}(n, R), O(n))_{0} \cong\left(\bigwedge \mathrm{gl}(n, R)^{*}\right)_{O(n)} \cong \wedge(\& / o(n))^{* O(n)},
$$

the set of elements in $\bigwedge(\mathrm{gl}(n, R) / o(n))^{*}$ invariant under the induced adjoint $O(n)$-actions on $\wedge(\mathrm{gl}(n, R) / o(n))^{*}$. The cohomology of $\left(\bigwedge \mathrm{gl}(n, R)^{*}\right)_{O(n)}$ is, by definition, $H(\operatorname{gl}(n, R), O(n))$; thus (7.1) is the map

$$
\Delta_{*}: H(\operatorname{gl}(n, R), O(n)) \cong H\left(W(\mathrm{GL}(n, R), O(n))_{0}\right) \rightarrow H(M) .
$$


Now,

$$
H(\operatorname{gl}(n, R), O(n)) \cong \bigwedge\left(x_{1}, x_{2}, \ldots, x_{n^{\prime}}\right)
$$

where $x_{1}, \ldots, x_{n}$ are the primitive elements which are transgressing to the Chern-classes $c_{1}, \ldots, c_{n}$ in $I(\mathrm{GL}(n, R))$ and $n^{\prime}=2[(n+1) / 2]-1$ is the largest odd integer $\leqslant n$. Hence we get an odd secondary class $\Delta_{*}\left(x_{i}\right) \in$ $H^{2 i-1}(M)$ in (7.1). If the given flat affine manifold $(M, \nabla)$ is a Riemannian manifold such that the connection $\nabla$ is the flat metric connection, then these invariants $\Delta_{*}\left(x_{i}\right)$ are zero.

Proposition 7.1. Let $M^{n}$ be a compact affine hyperbolic manifold. Then $\Delta_{*}\left(x_{1}\right)$ is a nonzero cohomology class.

Proof. See [8, Theorem 4.5].

Hence, Theorem 6.1 does produce nontrivial secondary classes even in the extreme situation.

In the second example, we modify an example in [9] to show that Theorem 6.2 also produces nontrivial secondary classes on the normal bundle of the nullity foliation.

Let $S^{2 r}=S O(2 r+1) / S O(2 r)$ be the $2 r$-sphere in $R^{2 r+1}$ where $S O(n)$ denotes the rotation group of degree $n$. The sphere $S^{2 r}$ is a Riemannian symmetric space with respect to the canonical $S O(2 r+1)$-invariant connection $\omega$ given by the $s o(2 r)$ component in the canonical decomposition ES $=s o(2 r+1)=s o(2 r)+\mathfrak{M}$. Here, an element $x \in \mathbb{Z}=\operatorname{so}(2 r)$ is identified with the element $\left[\begin{array}{l}x \\ 0\end{array}\right]$ in $s o(2 r+1)$, and $\mathfrak{M}$ is the subspace of all matrices of the form

$$
\left[\begin{array}{cc}
0 & v \\
-v & 0
\end{array}\right], \quad v=\left[\begin{array}{c}
v_{1} \\
v_{2} \\
\vdots \\
v_{2 r}
\end{array}\right] \in R^{2 r}
$$

Since so $(2 r+1)$ is semisimple, it follows from [13, Proposition 4.2] that the nullity distribution on $S^{2 r}$ is zero.

The isotropy representation $\rho: S O(2 r) \rightarrow \mathrm{gl}(\mathfrak{M})$ is given by

$$
\rho(a)\left[\begin{array}{cc}
0 & v \\
-v & 0
\end{array}\right]=\left[\begin{array}{cc}
0 & a v \\
-a v & 0
\end{array}\right]
$$

Therefore $\rho$ is given by the canonical representation of $S O(2 r)$ in $R^{2 r} \cong \mathfrak{M}$. It follows that $\rho: S O(2 r) \rightarrow S O(\mathfrak{M})=S O(2 r)$ is the identity representation. Hence, the principal $S O(2 r)$ bundle $P^{\prime}=S O(2 r+1) \rightarrow S^{2 r}$ is the orthogonal frame bundle of $S^{2 r}$. We next consider the pull-back of the bundle $P^{\prime}$, 


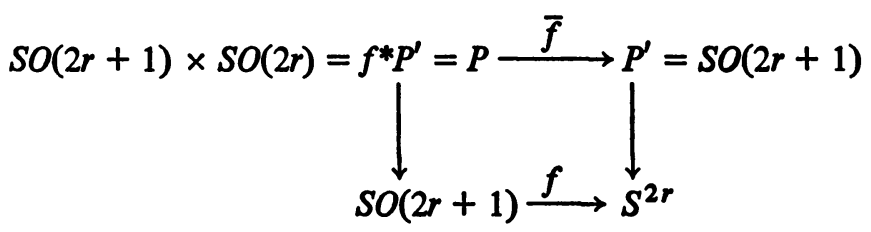

LEMMA 7.2. Let $\bar{\omega}=f^{*} \omega$ be the connection in $P$ defined by $\omega$. Let $\bar{\nabla}$ be the covariant derivation on $S O(2 r+1)$ corresponding to $\bar{\omega}$. Then the group $S O(2 r+1)$ with the affine connection $\bar{\nabla}$ has nullity foliation $N$ equal to the foliation defined by the submersion $f$.

Proof. Let $s$ be the cross-section defined by

$$
s(a)=(a, e) \text { for } a \in S O(2 r+1) \text {. }
$$

Then, it is easy to check that for $x, y \in \operatorname{so}(2 r+1)$, we have

$$
\begin{aligned}
\bar{R}(x, y) & =\left(s^{*} K_{\bar{\omega}}\right)(x, y)=s^{*}\left(d \bar{\omega}+\frac{1}{2}[\bar{\omega} \wedge \bar{\omega}]\right)(x, y) \\
& =\left(d \omega+\frac{1}{2}[\omega \wedge \omega]\right)(x, y)=-\omega[x, y]+[\omega(x), \omega(y)] .
\end{aligned}
$$

We need to show that $N$ is equal to $\operatorname{ker}\left(f^{*}\right)$. Let $x \in 3$. Then $\bar{R}(x, y)=$ $-\omega\left[x, y_{1}+y_{2}\right]+\left[x, \omega\left(y_{1}\right)+\omega\left(y_{2}\right)\right]$, where $y_{1} \in 3$ and $y_{2} \in \mathfrak{M}$ are such that $y=y_{1}+y_{2}$.

Therefore $\bar{R}(x, y)=-\omega[x, y]-\omega\left[x, y_{2}\right]+\left[x, y_{1}\right]$, since $\omega\left(y_{1}\right)=y_{1}$ and $\omega\left(y_{2}\right)=0$. Since $S O(2 r+1) / S O(2 r)$ is reductive, we have $\left[x, y_{1}\right] \in \mathcal{B}$ and $\left[x, y_{2}\right] \in \mathfrak{M}$. This implies that $\bar{R}(x, y)=0$. Hence $\operatorname{ker}\left(f^{*}\right) \subset N$. To show that $\operatorname{ker}\left(f^{*}\right)=N$, it suffices to show that if $x \in \mathfrak{M}$ and $\bar{R}(x, y)=0$ for all $y \in \mathbb{E}$, then $x=0$. Using the above argument, we have $\bar{R}(x, y)=-[x, y]=0$. But (S) $=\operatorname{so}(2 r+1)$ is semisimple, which implies that $x=0$. Q.E.D.

We note that $P=f^{*} P^{\prime}$ is the principal $S O(2 r)$ bundle of the normal bundle of the foliation defined by the submersions $f$. From the above lemma, it follows that $P$ is also the normal bundle of the nullity foliation $N$. Since $P$ is trivial, it has an $\{e\}$-reduction. Hence, from Theorem 6.2, the generalized characteristic homomorphism is of the form

$$
\Delta_{Q^{*}}: H^{\cdot}\left(W(S O(2 r))_{r}\right) \rightarrow H^{\cdot}(S O(2 r+1))
$$

where $2 r$ is the codimension of the nullity foliation $N$.

Proposition 7.3.

$$
\text { dimension } \operatorname{im}\left(\Delta_{Q^{*}}\right)= \begin{cases}2^{r-1}-2^{[r / 2]-1} & \text { for } r \text { even, } \\ 2^{r-1}+2^{[r / 2]} & \text { for } r \text { odd } .\end{cases}
$$

Proof. See [9, Theorem 6.52].

Hence, we conclude that there exist nontrivial secondary classes on the normal bundle $Q$ of the nullity foliation. 
REMARK. In [13], we observe that the study of nullity distributions can be regarded as a study of partial integrability of the underlying geometric structures. Thus, the generalized characteristic map $\Delta_{*}$ of Kamber and Tondeur in Theorem 3.1 can be used again to produce secondary classes for those geometric structures characterized by certain tensors $[13, \S 8]$.

\section{REFERENCES}

1. R. Bott, Lectures on characteristic classes and foliations, Lecture Notes in Math., vol. 279, Springer-Verlag, New York, 1972.

2. R. Bott and A. Haefliger, On characteristic classes of $\Gamma$-foliations, Bull. Amer. Math. Soc. 78(1972), 1039-1044. MR 46\#6370.

3. H. Cartan, Cohomologie réelle d'un espace fibré principal différentiable, Séminaire de Topologie Algébrique de l'Ecole Normale Supérieure, 1949/50, Exposés 19, 20, Math. Dept., M.I.T., Cambridge, Mass. MR 14, 670.

4. S. S. Chern and N. Kuiper, Some theorems on the isometric imbedding of compact Riemannian manifolds in Euclidean space, Ann. of Math. (2) 56 (1952), 422-430. MR 14, 408.

5. S. S. Chern and J. Simons, Some cohomology classes in principal fiber bundles and their application to Riemannian geometry, Proc. Nat. Acad. Sci. U.S.A. 68 (1971), 791-794. MR 43 \#5453.

6. C. Godbillon and J. Vey, Un invariant des feuilletages de codimension 1, C.R. Acad. Sci. Paris Sér. A-B 273(1971), A92-A95. MR 44\# 1046.

7. F. Kamber and $\mathrm{Ph}$. Tondeur, Cohomologie des algèbres de Weil relatives tronquées, $\mathrm{C} . \mathbf{R}$. Acad. Sci. Paris Sér. A-B 276 (1973), A459-A462; Algèbres de Weil semi-simpliciales, ibid., Al177-A1179; Homomorphisme caractéristique d'un fibré principal feuilleté, ibid., A1407-A1410; Classes caractéristiques derivés d'un fibré principal feuilleté, ibid., A1449-A1452. MR 47 \#9660, 9661, 9662; MR 50 \#4271.

8. , Characteristic invariants of foliated bundles, Manuscripta Math. 11 (1974), 51-89. MR 48 \#12556.

9. __ Non-trivial characteristic invariants of homogeneous foliated bundles, Ann. Ecole Norm. Sup. (to appear).

10. S. Kobayashi and K. Nomizu, Foundations of differential geometry, Vols. I, II, Interscience, New York, 1963, 1969. MR 27\#2945; 38\#6501.

11. R. Maltz, The nullity spaces of the curvature operator, Topologie et Géometrie Différentielle, Vol. 8, Centre Nat. Recherche Sci., Paris, 1966. MR 35 \#940.

12. J. S. Pasternack, Topological obstructions to integrability and the Riemannian geometry of smooth foliations, Thesis, Princeton Univ., 1970.

13. S. L. Tan, On nullity distributions, Trans. Amer. Math Soc 223 (1976), 323-335.

Department of Mathematics, University of Malaya, Kuala Lumpur, Malaysia 